Ugail H, Aggarwal A, Bakopoulos Y and Kotsios S (2008): "The Divider Set of Explicit Parametric Geometry", Cyberworlds 2008, IEEE Computer Society ISBN: 978-0-76953381-0, pp. 232-239. 


\section{The Divider Set of Explicit Parametric Geometry}

\author{
Hassan Ugail \\ School of Informatics \\ University of Bradford \\ Bradford BD7 1DP, UK \\ Email: h.ugail@bradford.ac.uk \\ Yannis Bakopoulos \\ Computational Applications Group \\ National Centre for Scientific Research, Demokritos \\ IAghia Paraskevi 153 10, POB 60228 \\ Athens, Greece \\ Email: ybak@dat.demokritos.gr
}

\author{
Akshai Aggarwal \\ School of Computer Science \\ University of Windsor, Windsor \\ Ontario, Canada \\ Email: akshaia@uwindsor.ca \\ Stelios Kotsios \\ Department of Economics \\ University of Athens \\ Pesmazoglou 8 \\ Athens 10559, Greece \\ Email: skotsios@econ.uoa.gr
}

\begin{abstract}
In this paper we describe a novel concept for classification of complex parametric geometry based on the concept of the Divider Set. The Divider Set is an alternative concept to maximal disks, Voronoi sets and cut loci. The Divider Set is based on a formal definition relating to topology and differential geometry. In this paper firstly we discuss the formal definition of the Divider Set for complex 3-dimensional geometry. This is then followed by the introduction of a computationally feasible algorithm for computing the Divider Set for geometry which can be defined in explicit parametric form. Thus, an explicit solution form taking advantage of the special form of the parametric geometry is presented. We also show how the Divider Set can be computed for various complex parametric geometry by means of illustrating our concept through a number of examples.
\end{abstract}

\section{Introduction}

Morphological shape descriptors such as skeletons, medial axis, cut loci and Voronoi sets are entities that can describe a complex object in a more compact form. It can be said that such morphological descriptors often have rich topologies that describe the underlying object in a general and canonical form. This enables these morphological descriptors to be utilised as useful and intuitive shape manipulation tools. A number of references discussing various morphological descriptors and their ability to naturally capture important shape characteristics of an object can be cited. e.g. $[18,15,6]$. For example, Blum [5] has suggested that the use of skeleton to be a powerful mechanism for representing the shape of two dimensional objects at a level higher than cell-enumeration. Here Blum puts forward a method for decomposing a shape into basic sub-shapes which can be associated with some primitive geometries.

When one is concerned with the representation of complex geometry within visual cyberworlds environments, such compact form of representation of complex geometric objects can be of importance. For example, in real time geometry modelling and animation scenarios the skeleton or the medial axis can play a vital role in enabling to efficiently manipulate a complex object. At the same time, it can also greatly enhance the animation aspects of an object within a visual cyberworlds environment. This may be especially true in the case where function based modelling and animation techniques are used whereby enhancing the real time applications within visual cyberworlds $[13,14]$.

In general when one is concerned with the morphological methods for extracting meaningful shape descriptors, they are often based on simple expansion and shrinking operations. These techniques have many practical applications. These include, image processing [9], shape similarity studies [5] and pattern recognition [8]. There are a number references on the medial axis. For comprehensive surveys the reader is referred to $[11,16]$. A number of methods for constructing medial axis or skeletons for polyhedral models and for free-form shapes have also been proposed. These include topological thinning [22], Euclidian distance transform[1] and the use of deformable snakes [12]. Exam- 
ples of practical algorithmic implementation of medial axis transforms can be found in, for example, [17, 19].

The most commonly used morphological description for geometric shapes is based upon the medial axis or the skeleton. The pioneering work of $\mathrm{H}$. Blum [5] describes the medial axis as a descriptor and classifier of shapes and figures. This concept has been established as the best defined and studied mathematical concept with reference to thinning and skeletonization of contours and shapes. From the various mathematical tools (e.g. maximal disks, cut loci, Voronoi sets [21, 7]), the maximal disk method seems to be the most well studied and applied, both in mathematical definition and properties and in applications.

The definition given for the medial axis by Blum can be mathematically described as follows.

Definition 1: Let $S$ be a closed contour in $\Re^{2}$. A closed disk $B$ is said to be maximal in $S$ if it is contained in $S$ and if $B \subset B^{\prime}$, where $B^{\prime}$ is another closed disk, also contained in $S$, then $B=B^{\prime}[21,7]$. The notion of maximal disks is based on the Euclidean metric,

$$
d_{E}(x, y)=\sqrt{\left(x_{1}-x_{2}\right)^{2}+\left(y_{1}-y_{2}\right)^{2}} .
$$

Taking the above definition of the medial axis as described above provides the description of an object which neatly describes the morphology and geometric characteristics of the underlying shape. Moreover, it can be highlighted that through the medial axis it is generally easier to identify the symmetries of the object. As mentioned earlier, other important properties of the media axis of a shape include its use in the intermediate representation of the object and its canonical general form that can be used to represent the object by a lower dimensional description. It is notable that many others have affirmed the flexibility of the medial axis and its ability to naturally capture important shape characteristics of an object $[18,15,6]$.

The idea of the Divider Set, which is the main discussion of this paper, relates to the concepts based on the description of the curvature of locally convex types [2] based on Huygens principles [20]. Such a description can serve as useful tools in other areas applicable to mathematics and physics such as knot theory, convexity, fluid flows as well as the study of differential equations, their solution propagations and description of singularities.

Hence this paper describes a concept which can be utilised for classification of complex geometry based on the concept we call as the Divider Set. The Divider Set is a novel alternative concept to maximal disks, Voronoi sets and cut loci, which is based on a formal definition based on topology and differential geometry. In particular in this paper we discuss how the Divider Set can be computed for complex parametric geometry which has an explicit form.
The paper is organised as follows. In Section 2 we introduce the idea behind the Divider Set along with formal definitions. In Section 3 we discuss how we can formulate solution techniques for computing Divider for parametric surfaces which can be described in a simpler form with an associated analytic expression. In Section 4 we discuss a series of examples where we show how the method described in Section 3 can be used to compute Divider Set for a variety of geometry defined in parametric form. Finally in Section 5 we conclude the paper and discuss possible extensions of the current work.

\section{The Divider Set of an Object}

If one undertakes a comparative analysis, the medial axis of a closed contour in $\Re^{2}$, in mathematical morphology, is probably the closest equivalent to the definition of the Divider Set in $\Re^{2}$ or $\Re^{3}$. The Divider Set in $\Re^{2}$ or $\Re^{3}$ is formally defined as follows.

Definition 2: Let $q \in S \subset \Re^{2}$ or $\Re^{3}$. Let $p \in \Re^{2}$ or $\Re^{3}$ define a direction $|p q|$ in $\Re^{2}$ or $\Re^{3}$ such that a closed ball $B(p,|p q|)$ having $p$ as the centre and $|p q|$ as the radius, in $\Re^{2}$ or $\Re^{3}$, with $q$ as the only point with $S$. i.e. $B(p,|p q|) \cup S=q$. Let $B_{c}\left(p_{c},\left|p_{c} q\right|\right)$ be the supremum of all balls $B(p,|p q|)$ with the above properties. Then, $B_{c}\left(p_{c},\left|p_{c} q\right|\right)$ is defined as the maximum contact ball of $S$ at $q \cdot \frac{1}{\left|p_{c} q\right|}=k_{c}$ is defined as the contact curvature of $S$ at $q$ and the locus of all $p_{c}$ 's for all $q$ 's of $S$ is called the Divider of $S$.

If one undertakes a comparative analysis between the formal definition of the Divider Set as described above and the formal definition of the maximal disks, Voronoi sets and cut loci, one can clearly see that there are some similarities between them. However, one should note that the definition of the Divider Set as presented above is a general definition which is based on the robust mathematical concepts and therefore provides specific advantages for description of the morphology. Thus, all definitions pertaining to medial axis, Voronoi sets, cut loci or any such concepts are replaced by a common definition expressed in a strict equation form.

It is important to highlight that the defining equations can be adapted to apply for curves [2] or disconnected sets of curves in $\Re^{2}$ or $\Re^{3}$, for isolated points, for surfaces in $\Re^{3}$ or for a mixture of the above. Furthermore, by a suitable change of the fundamental metric in the Euclidean plane and by considering a discretisation of the plane as in binary images, an entirely new form of the Divider Set can be developed and utilized in various applications.

In this paper the defining equations for the Divider Set is adapted to the three dimensional case whereby we consider surfaces $S \in \Re^{3}$ with some specific properties. The surfaces we consider are essentially parametric and should be, 
except for isolated points or curves, twice continuous and differentiable.

Given a surface $S \in \Re^{3}$ defined by the parametric form using two parameters $u$ and $v$ such that,

$$
S(u, v)=(x(u, v), y(u, v), z(u, v)),
$$

and given any values $u_{1}, v_{1}$ and $u_{2}, v_{2}$ of the defining parameters of $S$, we can define two points on $S$ such that,

$$
\begin{aligned}
& X_{1}\left(u_{1}, v_{1}\right)=\left(x_{1}\left(u_{1}, v_{1}\right), y_{1}\left(u_{1}, v_{1}\right), z_{1}\left(u_{1}, v_{1}\right)\right), \\
& X_{2}\left(u_{2}, v_{2}\right)=\left(x_{2}\left(u_{2}, v_{2}\right), y_{2}\left(u_{2}, v_{2}\right), z_{2}\left(u_{2}, v_{2}\right)\right) .
\end{aligned}
$$

Thus, taking three points $(x, y, z),\left(x_{1}, y_{1}, z_{1}\right),\left(x_{2}, y_{2}, z_{2}\right) \in S$ the calculation of the Divider involves solving the following equations.

$$
\begin{gathered}
\left(x_{1}-x\right) \frac{\partial x_{1}}{\partial u_{1}}+\left(y_{1}-y\right) \frac{\partial y_{1}}{\partial u_{1}}+\left(z_{1}-z\right) \frac{\partial z_{1}}{\partial u_{1}}=0 \\
\left(x_{2}-x\right) \frac{\partial x_{2}}{\partial u_{2}}+\left(y_{2}-y\right) \frac{\partial y_{2}}{\partial u_{2}}+\left(z_{2}-z\right) \frac{\partial z_{2}}{\partial u_{2}}=0 \\
\left(x_{1}-x\right) \frac{\partial x_{1}}{\partial v_{1}}+\left(y_{1}-y\right) \frac{\partial y_{1}}{\partial v_{1}}+\left(z_{1}-z\right) \frac{\partial z_{1}}{\partial v_{1}}=0 \\
\left(x_{2}-x\right) \frac{\partial x_{2}}{\partial v_{2}}+\left(y_{2}-y\right) \frac{\partial y_{2}}{\partial v_{2}}+\left(z_{2}-z\right) \frac{\partial z_{2}}{\partial v_{2}}=0 \\
\left(x_{1}-x\right)^{2}+\left(y_{1}-y\right)^{2}+\left(z_{1}-z\right)^{2} \\
=\left(x_{2}-x\right)^{2}+\left(y_{2}-y\right)^{2}+\left(z_{2}-z\right)^{2}
\end{gathered}
$$

Equations (5) - (9) can be also re-written in the following format,

$$
\begin{gathered}
x \frac{\partial x_{1}}{\partial u_{1}}+y \frac{\partial y_{1}}{\partial u_{1}}+z \frac{\partial z_{1}}{\partial u_{1}}=x_{1} \frac{\partial x_{1}}{\partial u_{1}}+y_{1} \frac{\partial y_{1}}{\partial u_{1}}+z_{1} \frac{\partial z_{1}}{\partial u_{1}} \\
x \frac{\partial x_{2}}{\partial u_{2}}+y \frac{\partial y_{2}}{\partial u_{2}}+z \frac{\partial z_{2}}{\partial u_{2}}=x_{2} \frac{\partial x_{2}}{\partial u_{2}}+y_{2} \frac{\partial y_{2}}{\partial u_{2}}+z_{2} \frac{\partial z_{2}}{\partial u_{2}} \\
x \frac{\partial x_{1}}{\partial v_{1}}+y \frac{\partial y_{1}}{\partial v_{1}}+z \frac{\partial z_{1}}{\partial v_{1}}=x_{1} \frac{\partial x_{1}}{\partial v_{1}}+y_{1} \frac{\partial v_{1}}{\partial v_{1}}+z_{1} \frac{\partial z_{1}}{\partial v_{1}} \\
x \frac{\partial x_{2}}{\partial v_{2}}+y \frac{\partial y_{2}}{\partial v_{2}}+z \frac{\partial z_{2}}{\partial v_{2}}=x_{2} \frac{\partial x_{2}}{\partial v_{2}}+y_{2} \frac{\partial y_{2}}{\partial v_{2}}+z_{2} \frac{\partial z_{2}}{\partial v_{2}} \\
x_{1}^{2}-x_{2}^{2}+y_{1}^{2}-y_{2}^{2}+z_{1}^{2}-z_{2}^{2} \\
=2\left(\left(x_{1}-x_{2}\right) x+\left(y_{1}-y_{2}\right) y+\left(z_{1}-z_{2}\right) z\right)
\end{gathered}
$$

One should note that for a given surface $S \in \Re^{3}$ the parameters $u_{1}$ and $v_{1}$ are known parameters spanning the surface $S$. The rest of the parameters $u_{2}, v_{2}, x, y, z, x_{1}$, $y_{1}, z_{1}, x_{1}, y_{2}, z_{2}$, can be calculated using the Equations (10) - (14). It is noteworthy that the Equations (5) - (9) are all linear in $x, y, z$, the Divider coordinates, while the Equations for $u_{2}$ and $v_{2}$ are highly non-linear. Furthermore, the solutions of the system must be examined to ensure that any balls with $(x, y, z)$ as a centre and,

$$
\begin{aligned}
R & =\sqrt{\left(x_{1}-x\right)^{2}+\left(y_{1}-y\right)^{2}+\left(z_{1}-z\right)^{2}} \\
& =\sqrt{\left(x_{2}-x\right)^{2}+\left(y_{2}-y\right)^{2}+\left(z_{2}-z\right)^{2}},
\end{aligned}
$$

as radii are indeed fully inscribed on the surface and do not contain points of it closer than their circumference.

\section{Method of Computation of the Divider Set}

In this section, based on the what we have discussed above, we show how we can apply the above methodology in order to compute the Divider Set for parametric surfaces which can be given in an explicit form.

The main difficulty in computing the Divider Set for a complex parametric surface arises due to the nature of the set of the Equations (10) - (14) to be considered. In particular, one should note that the Equations (14) is non-linear and therefore in general straightforward solution techniques do not exist to solve the system of Equations (10) - (14). In what follows, we describe a methodology based on exact solutions of the Equations (10) - (14) for the types of surfaces we are concerned here.

We assume a surface $S \in \Re^{3}$ can be defined by the parametric form using two parameters $u$ and $v$ such that $S(u, v)=(x(u, v), y(u, v), z(u, v))$. For the solution scheme proposed here we assume that the parametric surfaces can be described in the form,

$$
\begin{aligned}
& x(u, v)=f_{1}(u) \cos (n v), \\
& y(u, v)=f_{2}(u) \sin (n v),
\end{aligned}
$$

where $n$ is an integer and

$$
z(u, v)=f_{3}(u)
$$

Assuming our surface $S$ can be described in the above special form, it can be easily shown that the functions associated with Equations (10) - (14) are linearly independent. Therefore, the solution method based on the Cramer's rule through the computation of appropriate Wronskian for the related matrices can be used to solve the entire system of Equations (10) - (14). The procedures involved in solving the system is described below. 
The coefficient matrix of Equations (10) - (14) considered as a linear system $x, y, z$ as unknowns, is,

$$
\left(\begin{array}{ccc}
\frac{\partial x_{1}}{\partial u_{1}} & \frac{\partial y_{1}}{\partial u_{1}} & \frac{\partial z_{1}}{\partial u_{1}} \\
\frac{\partial x_{1}}{\partial v_{1}} & \frac{\partial y_{1}}{\partial v_{1}} & \frac{\partial z_{1}}{\partial v_{1}} \\
\frac{\partial x_{2}}{\partial u_{2}} & \frac{\partial y_{2}}{\partial u_{2}} & \frac{\partial z_{2}}{\partial u_{2}} \\
\frac{\partial x_{2}}{\partial v_{2}} & \frac{\partial y_{2}}{\partial v_{2}} & \frac{\partial z_{2}}{\partial v_{2}} \\
2\left(x_{1}-x_{2}\right) & 2\left(y_{1}-y_{2}\right) & 2\left(z_{1}-z_{2}\right)
\end{array}\right)
$$

The corresponding extended matrix, including the second part terms is,

$$
\left(\begin{array}{cccc}
\frac{\partial x_{1}}{\partial u_{1}} & \frac{\partial y_{1}}{\partial u_{1}} & \frac{\partial z_{1}}{\partial u_{1}} & A \\
\frac{\partial x_{1}}{\partial v_{1}} & \frac{\partial y_{1}}{\partial v_{1}} & \frac{\partial z_{1}}{\partial v_{1}} & B \\
\frac{\partial x_{2}}{\partial u_{2}} & \frac{\partial y_{2}}{\partial u_{2}} & \frac{\partial z_{2}}{\partial u_{2}} & C \\
\frac{\partial x_{2}}{\partial v_{2}} & \frac{\partial y_{2}}{\partial v_{2}} & \frac{\partial z_{2}}{\partial v_{2}} & D \\
2\left(x_{1}-x_{2}\right) & 2\left(y_{1}-y_{2}\right) & 2\left(z_{1}-z_{2}\right) & E
\end{array}\right)
$$

where

$$
\begin{gathered}
A=x_{1} \frac{\partial x_{1}}{\partial u_{1}}+y_{1} \frac{\partial y_{1}}{\partial u_{1}}+z_{1} \frac{\partial z_{1}}{\partial u_{1}}, \\
B=x_{1} \frac{\partial x_{1}}{\partial v_{1}}+y_{1} \frac{\partial y_{1}}{\partial v_{1}}+z_{1} \frac{\partial z_{1}}{\partial v_{1}}, \\
C=x_{2} \frac{\partial x_{2}}{\partial u_{2}}+y_{2} \frac{\partial y_{2}}{\partial u_{2}}+z_{2} \frac{\partial z_{2}}{\partial u_{2}}, \\
D=x_{2} \frac{\partial x_{2}}{\partial v_{2}}+y_{2} \frac{\partial y_{2}}{\partial v_{2}}+z_{2} \frac{\partial z_{2}}{\partial v_{2}}, \\
E=\left(x_{1}-x_{2}\right)^{2}+\left(y_{1}-y_{2}\right)^{2}+\left(z_{1}-z_{2}\right)^{2} .
\end{gathered}
$$

It may be assumed, without loss of generality, that Equations $(10),(11),(12)$ are linearly independent and may be used for the solution relative to $x, y$ and $z$. Here we outline the procedures involved.

Let $D_{x}$ be the determinant of the matrix,

$$
\left(\begin{array}{ccc}
x_{1} \frac{\partial x_{1}}{\partial u_{1}}+y_{1} \frac{\partial y_{1}}{\partial u_{1}}+z_{1} \frac{\partial z_{1}}{\partial u_{1}} & \frac{\partial y_{1}}{\partial u_{1}} & \frac{\partial z_{1}}{\partial u_{1}} \\
x_{1} \frac{\partial x_{1}}{\partial v_{1}}+y_{1} \frac{\partial y_{1}}{\partial v_{1}}+z_{1} \frac{\partial z_{1}}{\partial v_{1}} & \frac{\partial y_{1}}{\partial v_{1}} & \frac{\partial z_{1}}{\partial v_{1}} \\
x_{2} \frac{\partial x_{2}}{\partial u_{2}}+y_{2} \frac{\partial y_{2}}{\partial u_{2}}+z_{2} \frac{\partial z_{2}}{\partial u_{2}} & \frac{\partial y_{2}}{\partial u_{2}} & \frac{\partial z_{2}}{\partial u_{2}}
\end{array}\right) .
$$

Let $D_{y}$ be the determinant of the matrix,

$$
\left(\begin{array}{lll}
\frac{\partial x_{1}}{\partial u_{1}} & x_{1} \frac{\partial x_{1}}{\partial u_{1}}+y_{1} \frac{\partial y_{1}}{\partial u_{1}}+z_{1} \frac{\partial z_{1}}{\partial u_{1}} & \frac{\partial z_{1}}{\partial u_{1}} \\
\frac{\partial x_{1}}{\partial v_{1}} & x_{1} \frac{\partial x_{1}}{\partial v_{1}}+y_{1} \frac{\partial y_{1}}{\partial v_{1}}+z_{1} \frac{\partial z_{1}}{\partial v_{1}} & \frac{\partial z_{1}}{\partial v_{1}} \\
\frac{\partial x_{2}}{\partial u_{2}} & x_{2} \frac{\partial x_{2}}{\partial u_{2}}+y_{2} \frac{\partial y_{2}}{\partial u_{2}}+z_{2} \frac{\partial z_{2}}{\partial u_{2}} & \frac{\partial z_{2}}{\partial u_{2}}
\end{array}\right) .
$$

Let $D_{z}$ be the determinant of the matrix,

$$
\left(\begin{array}{lll}
\frac{\partial x_{1}}{\partial u_{1}} & \frac{\partial y_{1}}{\partial u_{1}} & x_{1} \frac{\partial x_{1}}{\partial u_{1}}+y_{1} \frac{\partial y_{1}}{\partial u_{1}}+z_{1} \frac{\partial z_{1}}{\partial u_{1}} \\
\frac{\partial x_{1}}{\partial v_{1}} & \frac{\partial y_{1}}{\partial v_{1}} & x_{1} \frac{\partial x_{1}}{\partial v_{1}}+y_{1} \frac{\partial y_{1}}{\partial v_{1}}+z_{1} \frac{\partial z_{1}}{\partial v_{1}} \\
\frac{\partial x_{2}}{\partial u_{2}} & \frac{\partial y_{2}}{\partial u_{2}} & x_{2} \frac{\partial x_{2}}{\partial u_{2}}+y_{2} \frac{\partial y_{2}}{\partial u_{2}}+z_{2} \frac{\partial z_{2}}{\partial u_{2}}
\end{array}\right) .
$$

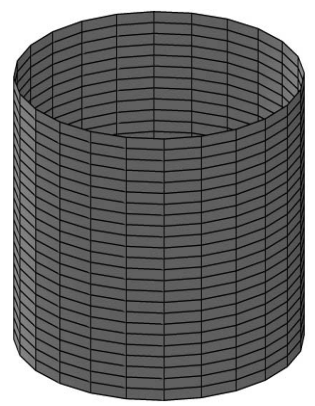

(a)

(b)

Figure 1. Computation of the Divider Set of a cylindrical surface. (a) Surface of the cylinder represented parametrically. (b) The corresponding Divider Set surface, which in this case is simply a straight line through the centre of the cylinder.

Finally let $D$ be the determinant of the matrix,

$$
\left(\begin{array}{lll}
\frac{\partial x_{1}}{\partial u_{1}} & \frac{\partial y_{1}}{\partial u_{1}} & \frac{\partial z_{1}}{\partial u_{1}} \\
\frac{\partial x_{1}}{\partial v_{1}} & \frac{\partial y_{1}}{\partial v_{1}} & \frac{\partial z_{1}}{\partial v_{1}} \\
\frac{\partial x_{2}}{\partial u_{2}} & \frac{\partial y_{2}}{\partial u_{2}} & \frac{\partial z_{2}}{\partial u_{2}}
\end{array}\right)
$$

Then Equations (10),(11),(12) yeild $x=D_{x} / D, y=$ $D_{y} / D$ and $z=D_{z} / D$. These values, depending on $u_{1}$ and $v_{1}$ which are known and on $u_{2}$ and $v_{2}$ which are unknown, can be put into Equations (13) and (14). These Equations will now contain $u_{2}$ and $v_{2}$ as unknowns, since $x, y$, and $z$ have been eliminated. Equations (13) and (14) may then be solved numerically.

\section{Examples}

In this section we show some examples where the above exact solution method can be utilised to compute the Divider Set for a number of surface geometry which can be described in parametric form. In order to compute the Divider Set for the examples shown here we have utilised the Maple package whereby we have taken advantage of mathematical operations and algorithms defined within Maple.

As a first example, we take a simple cylindrical shape. The cylinder is parametrically represented at $x=$ $\cos (v), y=\sin (v)$ and $z=u$. Fig. 1(a) shows the corresponding cylinder and Fig. 1 (b) shows the corresponding shape of the Divider Set which in this case is simply a line spanning along $u$ direction through the center of the cylinder.

As a second example, we take the geometry of an elliptic cylinder. The elliptic cylinder is parametrically represented at $x=A \cos (v), y=B \sin (v)$ and $z=u$ where $A \neq$ 


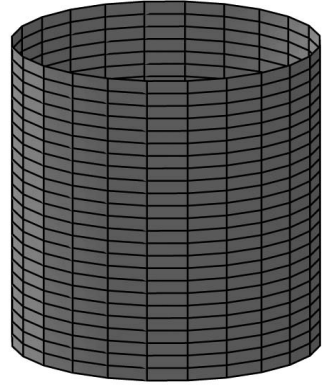

(a)

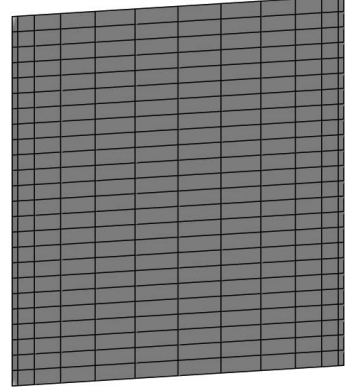

(b)
Figure 2. Computation of the Divider Set of an elliptic cylindrical surface. (a) Surface of the elliptic cylinder represented parametrically. (b) The corresponding Divider Set surface, which in this case is rectangular surface through the centre of the cylinder.

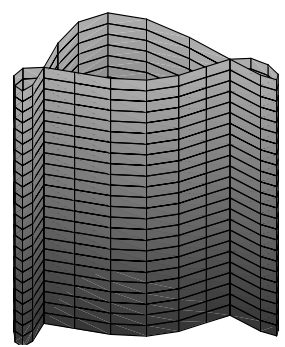

(a)

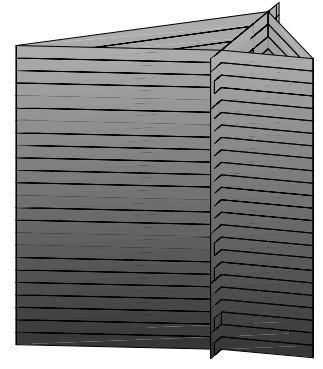

(b)
Figure 3. Computation of the Divider Set of a complex parametric patch. (a) Surface defined as analytic parametric functions. (b) The corresponding Divider Set surface.

$B$. Fig. 2(a) shows the corresponding elliptic cylinder and Fig. 2 (b) shows the corresponding shape of the Divider Set which in this case is a rectangular section spanning along $u$ direction passing through the centre of the cylinder.

As a third example, we take the geometry of a complex parametric patch defined by analytic parametric functions. The geometry in this case is defined as $x=A \cos (v)+$ $B \cos (2 v)+C \cos (3 v), y=D \sin (v)$ and $z=u$ where $A \neq$ $B$. Fig. 3(a) shows the corresponding parametric surface and Fig. 3 (b) shows the corresponding shape of the Divider Set.

The fourth example we describe is similar to that shown in the previous example. Here we take the geometry of a complex parametric patch defined by analytic parametric functions similar to the previous example. The geometry in this case is defined as $x=A \cos (v)+B \cos (2 v)+$

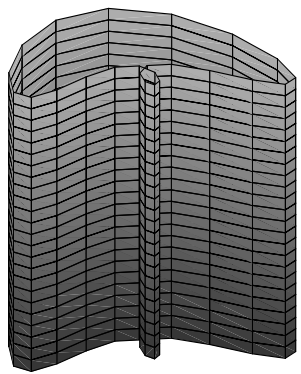

(a)

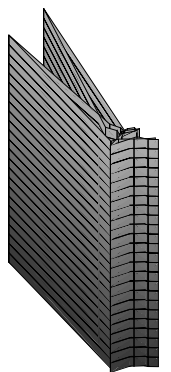

(b)
Figure 4. Computation of the Divider Set for a general parametric surface. (a) Surface defined parametrically using analytic functions. (b) The corresponding Divider Set surface.

$C \cos (3 v), y=D \sin (v)+E \sin (2 v)$ and $z=u$ where $A \neq B$. Fig. 4(a) shows the corresponding parametric surface and Fig. 4 (b) shows the corresponding shape of the Divider Set.

As a fifth example, we take the geometry represented by the harmonic partial differential equation (PDE). The harmonic PDE we use here is the standard Laplace equation,

$$
\left(\frac{\partial^{2}}{\partial u^{2}}+\frac{\partial^{2}}{\partial v^{2}}\right) X(u, v)=0 .
$$

Assuming the existence of periodic solutions for certain types of boundary conditions, the explicit solution of Equation (30) can be computed using separation of variables. Choosing the parametric region to be $0 \leq u \leq 1$ and $0 \leq v \leq 2 \pi$ the periodic boundary conditions can be expressed as,

$$
\begin{aligned}
& X(0, v)=P_{0}(v), \\
& X(1, v)=P_{1}(v),
\end{aligned}
$$

where the boundary conditions $P_{0}(v)$ and $P_{1}(v)$ define the edges of the surface patch at $u=0$ and $u=1$ respectively.

Then the explicit solution of Equation (30) can be written as,

$X(u, v)=A_{0}(u)+\sum_{n=1}^{\infty}\left[A_{n}(u) \cos (n v)+B_{n}(u) \sin (n v)\right]$

where

$$
\begin{gathered}
A_{0}=a_{00}+a_{01} u, \\
A_{n}=a_{n 1} e^{n u}+a_{n 2} e^{-n u}, \\
B_{n}=b_{n 1} e^{n u}+b_{n 2} e^{-n u},
\end{gathered}
$$

where $a_{n 1}, a_{n 2}, b_{n 1}$, and $b_{n 2}$ are vector-valued constants, whose values are determined by the imposed boundary conditions at $u=0$ and $u=1$. 


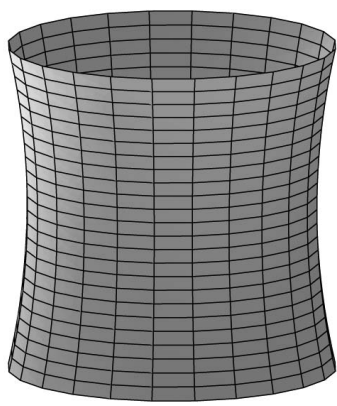

(a)

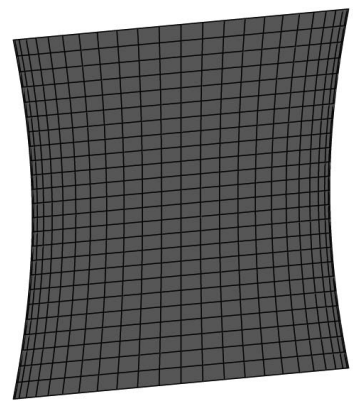

(b)
Figure 5. Computation of the Divider Set of a Harmonic surface. (a) A surface obtained by solving the Harmonic equation for a certain boundary conditions. (b) The corresponding Divider Set surface.

Fig. 5(a) shows a surface generated for the Laplace equation using the solution procedure described above. Here the boundary conditions through the Solution (33) can be written as,

$$
\begin{array}{r}
x(0, v)=A \cos (v), \\
y(0, v)=A \sin (v), \\
z(0, v)=0,
\end{array}
$$

$$
\begin{array}{r}
x(1, v)=B \cos (v), \\
y(1, v)=B \sin (v), \\
z(1, v)=1,
\end{array}
$$

where $A$ and $B$ are some real values. With these boundary conditions the corresponding surface is then obtained by determining the $A_{0}(u), A_{n}(u)$ and $B_{n}(u)$ and using Solution (33). Fig. 5 (b) shows the corresponding shape of the Divider Set for the harmonic surface.

As a final set of examples we show surfaces resulting to the solution from the Biharmonic equation based on the method of surface generation proposed by Bloor and Wilson [3]. The generating equation in this case is based on the Laplace equation (30) whereby the PDE is in the form,

$$
\left(\frac{\partial^{2}}{\partial u^{2}}+\frac{\partial^{2}}{\partial v^{2}}\right)^{2} X(u, v)=0 .
$$

Again, assuming the existence of periodic solutions for certain types of boundary conditions, the explicit solution

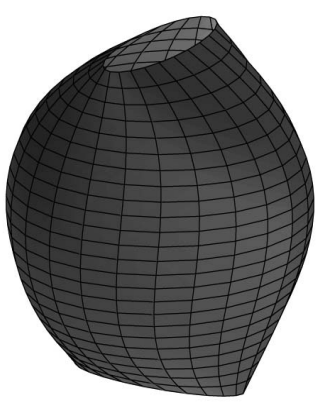

(a)

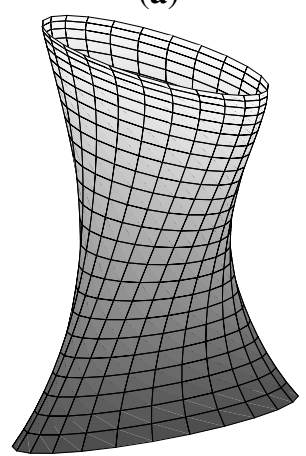

(c)

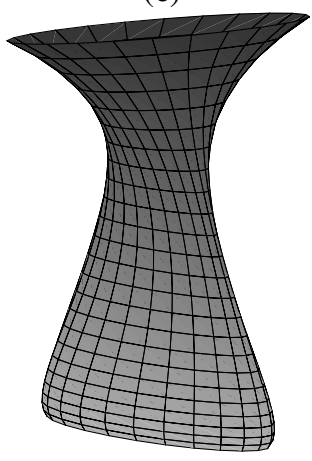

(e)

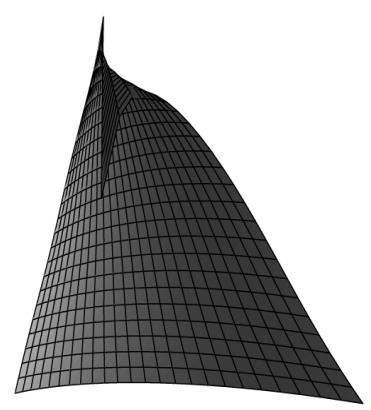

(b)

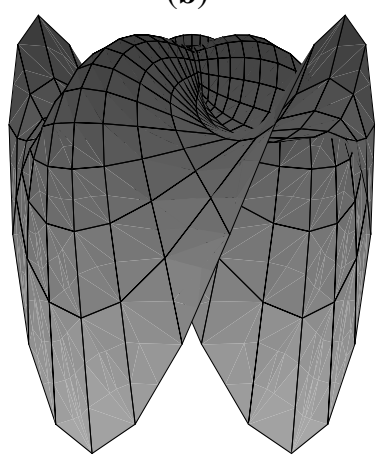

(d)

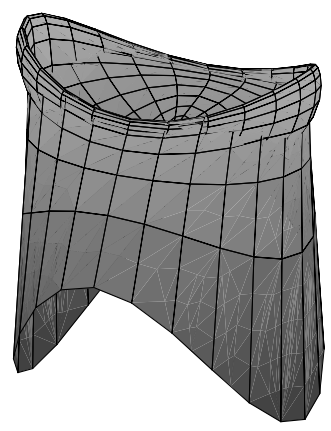

(f)
Figure 6. Computation of the Divider Set of a series of Biharmonic surfaces. (a, c, e)The surfaces obtained by solving the Biharmonic equation for a certain boundary conditions. (b, d, f) The corresponding Divider Set surfaces. 
of Equation (39) can be computed using separation of variables. Choosing the parametric region to be $0 \leq u \leq 1$ and $0 \leq v \leq 2 \pi$ the periodic boundary conditions can now be expressed as,

$$
\begin{aligned}
& X(0, v)=P_{0}(v), \\
& X(1, v)=P_{1}(v), \\
& X_{u}(0, v)=d_{0}(v), \\
& X_{u}(1, v)=d_{1}(v) .
\end{aligned}
$$

Here the boundary conditions $P_{0}(v)$ and $P_{1}(v)$ define the edges of the surface patch at $u=0$ and $u=1$ and the boundary conditions $d_{0}(v)$ and $d_{1}(v)$ define the derivatives at the edges of the surface patch at $u=0$ and $u=1$.

The explicit solution of the Biharmonic Equation (39) is also given by the Solution (33) where the vectors $A_{0}(u)$, $A_{n}(u)$ and $B_{n}(u)$ comprise of added terms to accommodate the higher order of the equation. i.e.,

$$
X(u, v)=A_{0}(u)+\sum_{n=1}^{\infty}\left[A_{n}(u) \cos (n v)+B_{n}(u) \sin (n v)\right],
$$

where

$$
\begin{gathered}
A_{0}=a_{00}+a_{01} u+a_{02} u^{2}+a_{03} u^{3}, \\
A_{n}=a_{n 1} e^{n u}+a_{n 2} e^{-n u}+a_{n 3} u e^{n u}+a_{n 4} u e^{-n u}, \\
B_{n}=a_{n 1} e^{n u}+a_{n 2} e^{-n u}+a_{n 3} u e^{n u}+a_{n 5} u e^{-n u},
\end{gathered}
$$

where $a_{n 1}, a_{n 2}, a_{n 3}, a_{n 4}, b_{n 1}, b_{n 2}, b_{n 3}$, and $b_{n 4}$ are vectorvalued constants, whose values are determined by the imposed boundary conditions at $u=0$ and $u=1$. Detailed description of this solution scheme can be found in [3].

Figs. 6(a)(c)(e) shows a series of surfaces generated through the Biharmonic equation using the solution procedure described above. Here the boundary conditions for the surface can be written as,

$$
\begin{array}{r}
x(0, v)=A \cos (v), \\
y(0, v)=A \sin (v), \\
z(0, v)=0, \\
\\
x(1, v)=B \cos (v), \\
y(1, v)=B \sin (v), \\
z(1, v)=1, \\
\\
x_{u}(0, v)=C \cos (v), \\
y_{(}(0, v)=C \sin (v), \\
z_{u}(0, v)=0,
\end{array}
$$

$$
\begin{array}{r}
x_{u}(1, v)=D \cos (v), \\
y_{u}(1, v)=D \sin (v), \\
z_{u}(1, v)=0,
\end{array}
$$

where $A, B, C, D$ are some real values.

With these boundary conditions the corresponding surface is then obtained by determining the $A_{0}(u), A_{n}(u)$ and $B_{n}(u)$ and using an extended version of the Solution (33). Fig. $6(\mathrm{~b})(\mathrm{d})(\mathrm{f})$ shows the corresponding shapes of the Divider Sets for the Biharmonic surfaces.

\section{Conclusion}

In this paper we have described a concept for classification of complex parametric geometry based on the concept of the Divider Set. The Divider Set is a novel alternative concept to maximal disks, Voronoi sets and cut loci, based on a formal definition relating to topology and differential geometry. In this paper we have shown how the Divider Set can computed for surfaces described in parametric form. We have described how an explicit solution form can be used to solve the related system defining the Divider Set. A number of examples are discussed in order to show the feasibility of the Divider for shape description.

There a number of extensions to this work. In this paper, we have described the methods of computing the Divider Set for parametric surfaces. While parametric surfaces are applicable to a number of areas there are situations where surfaces or solids cannot be defined in parametric form. Thus, the solution methods we have discussed would need further developments. Particular cases of solutions can be developed for particular types of surfaces (e.g. PDE surfaces) where the computation of the Divider Set can be made more efficient. Another area to extend this work is to study the Divider Set for discrete cases. This has applications in situations where the geometry and images in question are available in discrete form. Our ongoing and future work in this topic aims to extend our research efforts in these areas.

\section{References}

[1] C. Arcelli and G. Sanniti di Baja, Ridge Points in Euclidean Distance Maps, Pattern Recognition Letters, 13(4), 37-243, 1992.

[2] Y. Bakopoulos and P. C. Stavrinos, The Generalized Curvature of Locally Convex Type, ELEFTERIA (S.P. Zervos ed.), 4B, 267-280, 1986.

[3] M. I. G. Bloor and M. J. Wilson. Using Partial Differential Equations to Generate Freeform Surfaces, Computer-Aided Design, 22, 202-212, 1990. 
[4] M. I. G. Bloor, and M. J. Wilson. it An analytic Pseudo-Spectral Method to Generate a Regular 4-sided PDE surface Patch, Computer Aided Geometric Design, 22(3), 203-219, 2005.

[5] H. Blum, A transformation for extracting new descriptions of shape, In W. Wathen-Dunn, editor, Models for the Perception of speech and Visual Form, MIT Press, 362-380, 1967.

[6] J. D. Boissonnat, Geometric Surfaces for 3Dimensional Shape Representation, ACM Transactions on Graphics, 3(4), 244-265, 1984.

[7] H. I. Choi, S. W. Choi and H. P. Moon, Mathematical Theory of Medial Axis Transform, Pacific Journal of Mathematics, 181(1) (1997) 57-88

[8] R. Duda and P. Hart, Pattern Classification and Scene Analysis, Wiley-Interscience, New York, (1973).

[9] D. S. Fritsch, S. M. Pizer, B. S. Morse, D. H. Eberly and A. Liu, The Multiscale Medial Axis and Its Applications in Image Registration, Pattern Recognition Letters, 15(5), 445-452, 1994.

[10] N. Kalouptsidis, Signal Processing Systems, WileyIntersience, 1997.

[11] L. Lam, S.-W. Lee and C. Suen. Thinning Methodologies - A Comprehensive Survey, IEEE Transactions on Pattern Analysis and Machine Intelligence, 14, 869885, 1992.

[12] F. Leymarie and M. D. Levine, Skeleton from Snakes, Progress in Image Analysis and Processing, World Scientific Singapore, 1990.

[13] Q. Liu and A. Sourin, Function-Based Representation of Complex Geometry Appearance, in Proceedings of ACM Web3D '05, 123134, 2005.

[14] Q. Liu and A. Sourin, Function-Defined Shape Metamorphoses in Visual Cyberworlds, The Visual Computer, 22, 977-990, 2006.

[15] L. R. Nackman and S. M. Pizer. Three-Dimensional Shape Description using Symmetric Axis Transform, IEEE Transactions on Pattern Analysis and Machine Intelligence, 7(2), 187-202, 1985.

[16] R. Ogniewicz, Discrete Voronoi Skeletons, HartungGorre Verlag, Konstanz, Germany, 1993.

[17] J. O'Rourke, Computational Geometry in C, Cambridge University Press, New York, 1994.
[18] N. M. Patrikalakis and H. N. Gursoy, Shape Interrogation by Medial Axis Transform, In: Ravani, B. (ed.): Advances in Design Automation: ComputerAided Computational Design, ASME 1,77-88, 1990.

[19] T. Pavlidis, Algorithms for Graphics and Image Processing, Computer Science Press, Rockville MD, 1982.

[20] S. J. Ruuth and B. Merriman, Convolution-Generated Motion and Generalized Huygens' Principles for Interface Motion, SIAM Journal on Applied Mathematics, 60(3), 868-890, 2000.

[21] E. C. Sherbrooke, N. M. Patrikalakis and F. Wolter, Differential and Topological Properties of Medial Axis Transforms, Graphical Models and Image Processing, 58(6), 574-592, 1996.

[22] Y. F. Tsao and K. S. Fu, A Parallel Thinning Algorithm for 3-D Pictures, Computer Graphics Image Process, 17, 315-331, 1981. 